\title{
Mobile Tele-Echography: User Interface Design
}

\author{
Cristina Cañero, Nikolaos Thomos, Student Member, IEEE, George A. Triantafyllidis, George C. Litos, and \\ Michael Gerassimos Strintzis, Fellow, IEEE
}

\begin{abstract}
Ultrasound imaging allows the evaluation of the degree of emergency of a patient. However, in some instances, a well-trained sonographer is unavailable to perform such echography. To cope with this issue, the Mobile Tele-Echography Using an Ultralight Robot (OTELO) project aims to develop a fully integrated end-to-end mobile tele-echography system using an ultralight remote-controlled robot for population groups that are not served locally by medical experts.

This paper focuses on the user interface of the OTELO system, consisting of the following parts: an ultrasound video transmission system providing real-time images of the scanned area, an audio/video conference to communicate with the paramedical assistant and with the patient, and a virtual-reality environment, providing visual and haptic feedback to the expert, while capturing the expert's hand movements. These movements are reproduced by the robot at the patient site while holding the ultrasound probe against the patient skin. In addition, the user interface includes an image processing facility for enhancing the received images and the possibility to include them into a database.
\end{abstract}

Index Terms-Haptic interfaces, telemedicine, ultrasound imaging, video compression, virtual reality.

\section{INTRODUCTION}

$\mathbf{M}$ OBILE interconnectivity and telemedicine are important issues in achieving effectiveness in health care, since medical information can be transmitted faster and physicians can make diagnoses and treatment decisions faster. A context where telemedicine can be of great help is the one of ultrasound imaging. While this technique allows the evaluation of the degree of emergency of a patient, many small medical centers, usually isolated, or rescue vehicles do not have the well-trained sonographers to perform such echography. To cope with this issue, the Mobile Tele-Echography Using an Ultralight Robot (OTELO) project aims to develop a fully integrated end-to-end mobile tele-echography system using an ultralight remote-controlled robot, for population groups that are not served locally by medical experts.

Recently, many telemedicine systems related with ultrasound imaging have been proposed. A laparoscopic system in which an expert tele-operates a robot by holding an ultrasound probe is presented in [1]. Simultaneous videoconference of two experts [2], one located at the patient side and the other at a distant

Manuscript received August 29, 2003; revised June 4, 2004. This work was supported by the European Union Project IST-2001-32516 OTELO.

C. Cañero, G. A. Triantafyllidis, and G. C. Litos are with the Informatics and Telematics Institute, Centre of Research and Technology Hellas, Thessaloniki 57001, Greece.

N. Thomos and M. G. Strintzis are with the Informatics and Telematics Institute, Centre of Research and Technology Hellas, Thessaloniki 57001, Greece. They are also with the Electrical and Computer Engineering Department, Aristotle University of Thessaloniki, Thessaloniki 54124, Greece.

Digital Object Identifier 10.1109/TITB.2004.840064 location, has been used for distant diagnosis. Another system has been developed by TeleInVivo [3], in which the echography was performed by a clinical expert standing near to the patient and then the acquired ultrasound data was sent via satellite to a database station for processing and to reconstruct a three-dimensional (3-D) representation of anatomical region of interest.

The OTELO system is designed to guarantee a reliable echographic diagnosis in an isolated site that is not served locally by medical experts. An expert sonographer located at the master medical center performs the echographic diagnosis, while at the isolated medical center, only a paramedical assistant is needed. At the master station, the clinical expert controls and tele-operates the distant robot by holding a fictive probe. The robot holds an ultrasound probe and reproduces, in real-time, the motion of a fictive probe held by the expert. Ultrasound images are sent from the patient's site to the expert's site. A virtual 3-D rendering of the patient and the position of the ultrasound probe are also provided to the expert. Hence, this design allows the expert to perform the examination as if he/she was virtually present at the patient site.

This paper focuses on two specific and strongly linked components of the OTELO tele-echography system which are very important for the overall efficiency of the system. Specifically, this paper proposes the architecture for the ultrasound image communication, as well as the user interface for the visualization of the ultrasound images and the remote monitoring and virtual-reality controlling of the mobile ultrasound examination.

The paper is organized as follows. In Section II, an overall description of the OTELO system is presented. Section III presents the system setup, while Section IV describes in detail the ultrasound compression scheme, the required bandwidth and image quality to assure diagnostic usability. Section $V$ presents the proposed interface for the tele-echography examination, while the basic implementation issues are shown in Section VI. The paper ends with conclusions and foreseen improvements.

\section{Overall DESCRIPTION OF THE SYSTEM}

The OTELO system provides a fully integrated end-to-end communication between patient and expert stations. The overall system consists of the following:

- A six degree-of-freedom (DOF) light robot with an ultrasound probe is operated by the paramedical assistant at the patient site. More details relating the robot design can be found in [4].

- An interface which displays the ultrasound images, enables robot controlling by the medical expert, and provides force feedback information at the expert site. 
- A communication link (satellite or terrestrial) which allows information exchange between the two sites (i.e., ambient audio and video, ultrasound images, and robot control data).

The paramedical assistant positions the robot to the patient's body according to the expert's instructions received through the videoconference link and the virtual-reality environment. Right after the correct robot placement, the examination session can be started. The fictive probe captures the movements of the expert hand, which are sent to the patient station. The robot reproduces the expert movements, measures the force exerted on the patient skin, and performs the echography examination. The ultrasound images and the force information are sent in real time to the expert. This information is required for the efficient distant robot controlling, in order to examine different anatomical regions and search for the region of interest. The interactive control is important, since the physician searches for the best possible ultrasound image to facilitate diagnosis.

In addition, a virtual 3-D rendering of the patient and the position of the ultrasound probe is provided to the expert. Hence, the expert is able to "feel" the patient as if he/she were present at the patient site. Moreover, a videoconference link enables patient-expert direct communication, while it makes the patient feel more comfortable.

A lossy video compression algorithm is employed for realtime video transmission due to bandwidth limitations. Whenever the expert needs a still image of a high quality (for better inspection and storage into the patient's record), a full-size losslessly coded still image is sent to the expert.

One of the main objectives of the system is to provide the expert with the sense of telepresence. To this end, the following tools are provided for the tele-echography examination:

- A virtual-reality environment. The patient's body and the robot are illustrated by 3-D models. Using the mouse, the expert can reposition and rotate the robot and patient models or zoom in a selected area. This rendering is displayed also at the patient site. Thus, the paramedical assistant is continuously aware of the required exact positions for the patient's body and the robot.

- Ultrasound image transmission. The ultrasound images acquired at the patient site are compressed and sent to the expert station in real time. This allows the expert to perform the echography examination and locate lesions and other problems.

- Audio/video conference. The expert is able to communicate in real time with both patient and the paramedical assistant through an audio/video conference link. The videoconference link is used at the beginning of the examination to provide more comfort to the patient. During the examination, the ambient video is transmitted whenever high bandwidth is available.

\section{System SETUP}

The system setup and connection protocol have been designed to be functional and fast, since these operations are performed quite frequently. At the expert station, the expert sets up the virtual probe, while the paramedical assistant at the patient station should also perform the robot setup. Prior the examination, the paramedical assistant sends a request for connection to the expert station and then waits for confirmation. If the expert station is serving, the expert accepts the connection and a message of connection acceptance is sent to the patient station.

When the connection is established, the patient data (patient's name, age, weight, height, etc.) are requested. In addition, the paramedical assistant selects among the available 3-D models (man/woman, pregnant, child, etc.) the best fitting model. These data are sent to the expert station, where they are displayed to the expert and stored for subsequent use. Then, the audio and video conference link between the expert and patient station is initiated.

The expert using the virtual-reality environment positions the robot model on the patient's model. Then the desired position and orientation are transmitted to the patient station where they are visualized in a virtual-reality environment to assist the paramedical assistant in positioning the robot on the patient's body. Using the ambient video at the patient station and the audio link, the expert can directly monitor the robot placement When the robot is correctly placed, the examination procedure can be started. The patient station sends the ultrasound video and the robot status data, while the expert station sends robot commands for reproduction of expert hand movements. The whole procedure is summarized in Fig. 1.

\section{Ultrasound IMAGE SEQUENCE TRANSMISSION}

The ultrasound images are acquired at the patient station at a resolution of $768 \times 570$. However, due to bandwidth restrictions, the chosen image size for real-time video transmission is $352 \times 288$, and the images are sent using a lossy video compression algorithm.

The selection of high performance real time video coders is crucial for medical data compression. Recently, a wavelet-based coder endowed with the property of fine granular scalability [5] has been proposed for compression of medical video sequences. Conventional compression techniques like ITU standards H.263 [6] and H.264 [7] can also be used for medical purposes since they offer almost visual lossless quality, while saving large amounts of data.

The above video coding schemes were experimentally evaluated for the transmission of ultrasound video sequences with a frame resolution of $352 \times 288$. The H.263 coder has been selected because of the low computational cost that it is in accordance to the video transmission needs set by the OTELO project.

Expert evaluation has shown that $36 \mathrm{~dB}$ and $5 \mathrm{fps}$ are considered as the minimum requirements for an efficient diagnosis. In Table I, results are reported for two abdominal sequences. Both sequences are encoded using the selected H.263 coder. The resultant peak signal-to-noise ratio (PSNR) values (in terms of decibels) and the corresponding actual frame rate in frames per second are presented. According to the evaluation $26 \mathrm{~kb} / \mathrm{s}$ seems to be adequate for the first sequence, while the second ultrasound sequence needs for acceptable quality $104 \mathrm{~kb} / \mathrm{s}$. This bandwidth variations are due to different compression ratios, 


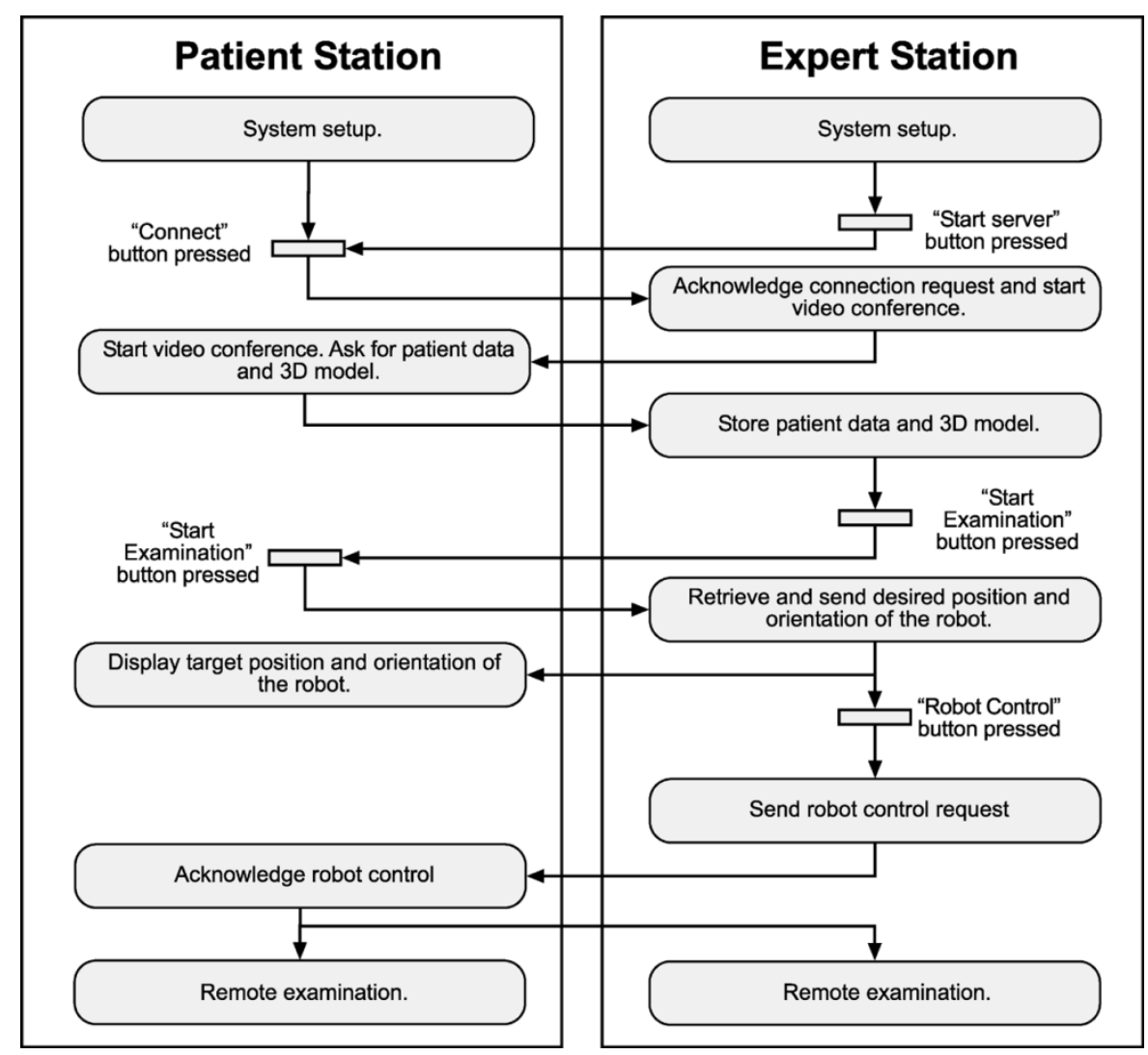

Fig. 1. Summary of system setup and connection protocol.

TABLE I

PSNR AND FRAME RATE OF H.263 CODER FOR Two ABDOMINAL SEQUENCES

\begin{tabular}{c|c|c|c|c}
\hline \multirow{2}{*}{$\begin{array}{c}\text { US Bit Rate } \\
(\text { Kbps })\end{array}$} & \multicolumn{2}{|c|}{ sequence 1 } & \multicolumn{2}{c}{ sequence 2 } \\
\cline { 2 - 5 } & PSNR & Frame Rate & PSNR & Frame Rate \\
\hline \hline 26 & 37.01 & 7.69 & 32.84 & 11.11 \\
\hline 40 & 37.2 & 12.82 & 33.26 & 12.04 \\
\hline 104 & 40.43 & 13.00 & 37.31 & 11.95 \\
\hline 232 & 44.89 & 14.12 & 38.16 & 11.59 \\
\hline
\end{tabular}

since the compression ratio depends on the quality of the ultrasound probe and the experience of the paramedical assistant. After numerous tests, we concluded that $128 \mathrm{~kb} / \mathrm{s}$ meets both quality and frame rate conditions to assure diagnostic usability of all ultrasound sequences.

\section{TELE-EChOGRAPHY EXAMINATION INTERFACE}

During the remote examination procedure, the medical doctor can switch between the robot placement and robot control.

\section{A. Robot Placement}

The window shown during the robot placement procedure is illustrated in Fig. 2. A virtual environment enables the expert to select the desired position and orientation of the robot using the mouse. This 3-D information is sent to the patient station, where it is rendered too. Therefore, the paramedical assistant is aware of the desired exact positioning of the robot. A high-quality (common interchange format (CIF) size) ambient video is transmitted (using an H.263 codec) allowing the expert to monitor the robot positioning. When the robot is correctly positioned,

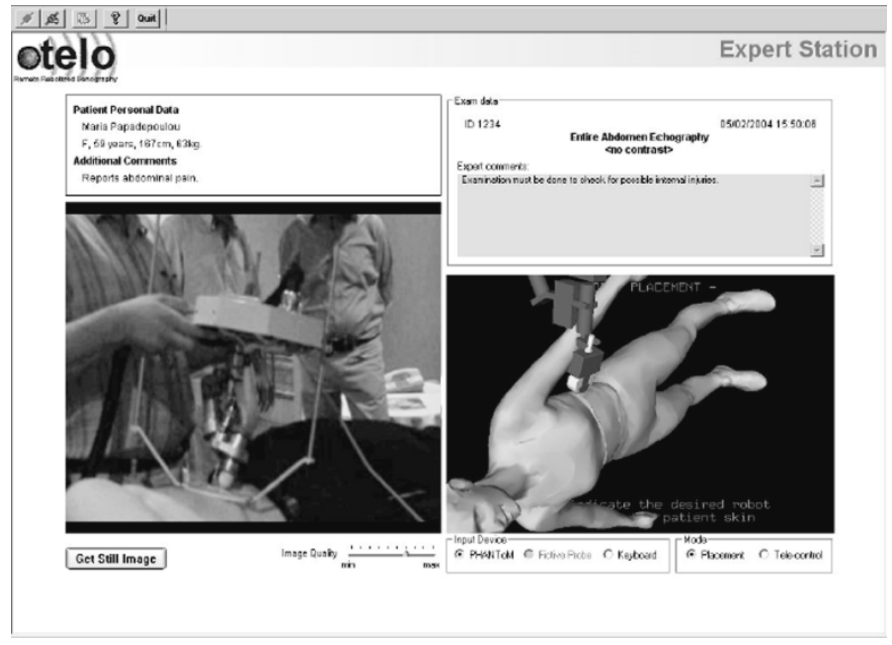

Fig. 2. Displayed window at the expert station during robot placement.

the expert can gain control of the robot by activating the "Robot Control" radio button.

\section{B. Robot Control}

The window for robot control is depicted in Fig. 3. The input device tracks the position and orientation of the expert hand, which are sent to the patient station and reproduced by the robot. A virtual environment renders the patient's body and the robot, allowing the expert to have a better insight of where the ultrasound probe is. The expert receives CIF size ultrasound images from the patient station and quarter CIF for the ambient video. 


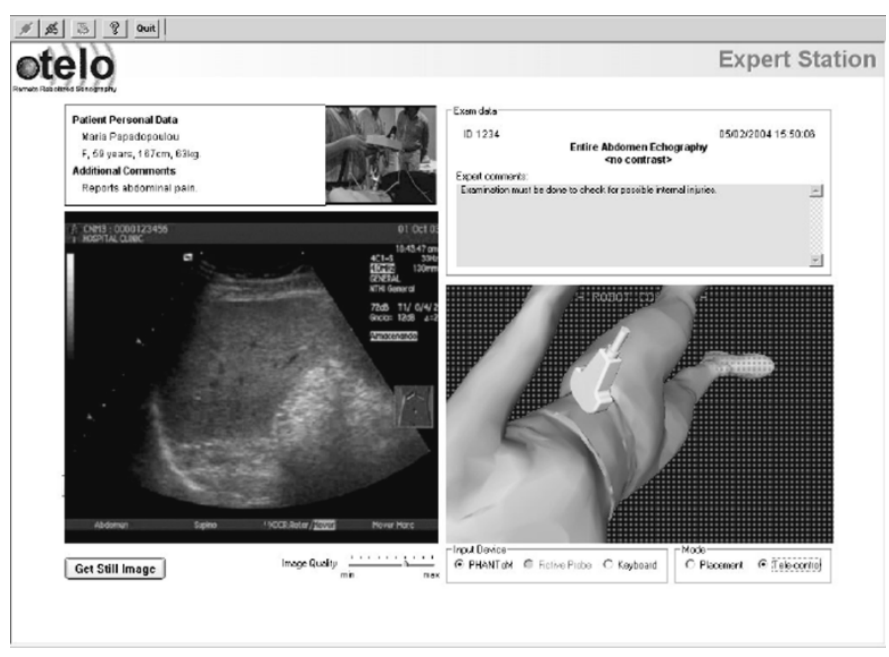

Fig. 3. Displayed window at the expert station during robot control.
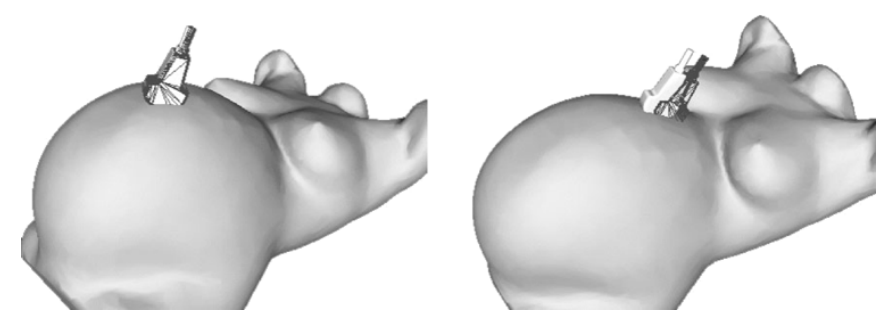

Fig. 4. Target position of the ultrasound probe is rendered as a wireframe, while the real position as a solid model.

However, in case of a weak communication link, all the available bandwidth is assigned to the ultrasound video and control data.

Telerobotic applications usually suffer from delays due to heterogeneous communication channels and mechanical limitations. During testing, one of the main complaints of the medical experts was the delay between the movement of the fictive probe and the actual movement of the robot at the patient station. To alleviate this problem, the expert moved the fictive probe very slowly, allowing the robot to follow the target position. However, the expert had no feedback about whether the probe is actually following his/her movements. Two possible solutions are developed to provide the expert with such feedback: a visual-feedback and a force-feedback approach. In both cases, the current position and orientation of the ultrasound probe is sent to the Expert Station.

- In the visual-feedback approach, the target position of the ultrasound probe (i.e., intended position of the robot) is rendered as a black wireframe, and the real one as a white solid realistic model (see Fig. 4). Therefore, the expert has insight whether the input device is being moved too fast, and about the actual real position of the robot at each moment.

- The force-feedback consists of a new added force effect to the haptic environment that attracts the fictive probe to the current position of the robot. Thus, whenever the

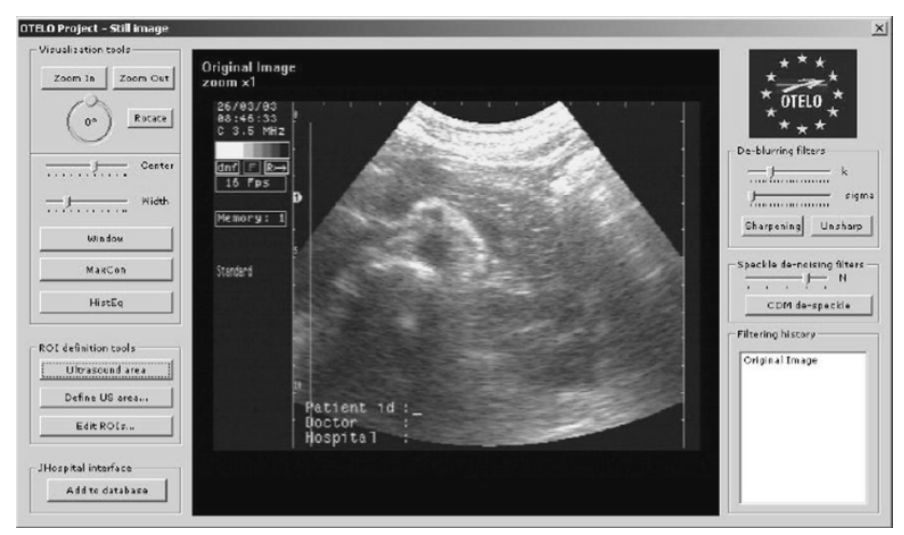

Fig. 5. Ultrasound still image interface.

expert moves the probe too fast, he/she will experience a proportional resistance against this movement.

The expert adjusts the image quality using the slide bar in the interface to assure efficient diagnosis. In this way, the expert compromises the ultrasound image quality and frame rate for a given bandwidth. Although the obtained video quality can be adequate for the examination, the experts may require losslessly coded images for their final diagnosis and for storage into the patient's record. Thus, a full-size still image can be requested by the expert, so an image is captured at the patient station, losslessly compressed using JPEG2000-LS [8] at the patient station and sent to the expert. When the image is received, a new window is displayed (Fig. 5), granting access to the medical database interface and providing several image processing functionalities.

The following image processing functions are available:

1) Zooming and rotation: Image to a higher resolution can make the image appear easier to interpret, especially at small details. In some cases, image rotation can further improve the image comprehension by the expert.

2) Histogram operations: The user is able to perform three different histogram transformations: gray-scale windowing, contrast maximization, and histogram equalization.

3) Deblurring filters: Image sharpening makes slightly blurry pictures more detailed. This is useful after the application of a denoising filter, since some of such filters have the colateral effect of blurring the edges.

4) Speckle noise reduction: Ultrasound images are corrupted by speckle noise, which is often modeled as a multiplicative process. Speckle noise often prevents experienced radiologists from drawing useful conclusions, and therefore, should be filtered out without destroying important features.

\section{IMPLEMENTATION ISSUES}

The applications have been implemented using Visual $\mathrm{C}++$ and the MFC library. The ultrasound signal and the ambient video are acquired using DirectShow, and the video signals are 
transmitted using the selected video codec (H.263). The packages are sent using transmission control protocol/Internet protocol link to the client where the proper video decoder provides the video signal, which is displayed on the corresponding window. The audio conference has been implemented using the open source voice over network API HawkVoiceDI [9]. In order to create the record that is sent to database, the ZLib library [10] has been selected. For the virtual examination, OpenGL has been employed for the 3-D model rendering.

\section{CONCLUSION}

An implementation for the OTELO tele-echography system user interface has been proposed in this paper. It allows the expert and the paramedical assistant to efficiently set and control the positioning of the robotic system and perform the teleechography act. Specifically, an appropriately designed 3-D environment facilitates positioning and robot control. Ultrasound and ambient video communication and audio conference links are also employed for the examination procedure using efficient compression algorithms. An analysis of the required bandwidth and the received image quality was presented in this paper. The next foreseen step is, therefore, to perform extensive clinical tests of the whole system for various bandwidth scenarios to evaluate the best configuration for each case of the ultrasound examinations to achieve diagnostic usability.

\section{REFERENCES}

[1] D. D. Cunha and P. Gravez, "The MIDSTEP system for ultrasound guided remote telesurvery," in 20th Annu. Intelligent IEEE Engineering in Medicine and Biology Soc., 1998, pp. 1266-1269.

[2] E. Degoulange, S. Boudet, J. Gariepy, F. Pierrot, L. Urbain, J. Megnier, E. Dombre, and P. Caron, "Hippocrate: An intrinsically safe robot for medical application," in IEEE/RSJ Int. Conf. Intelligent Robot and Systems, Oct. 1998, pp. 959-964.

[3] G. Kontaxakis, S. Walter, and G. Sakas, "EU-TeleInViVo: An integrated portable telemedicine workstation featuring acquisition, processing and transmission over low-bandwidth lines of 3D ultrasound volume images," in Proc. IEEE EMBS Int. Conf. Inform. Technol. Applicat. Biomed. (ITAB 2000), Arlington, VA, Nov. 2000, pp. 158-163.

[4] L. Al Bassit, G. Poisson, and P. Vieyres, "Kinematics of a 6DOF robot for tele-echography," in 11th Int. Conf. Advanced Robotics (ICAR 2003), Coombra, Portugal, Jun. 30-Jul. 3, 2003.

[5] K. E. Zachariadis, N. V. Boulgouris, N. Thomos, G. A. Triantafyllidis, and M. G. Strintzis, "Wavelet-based communication of medical image sequences," in Int. Workshop Enterprise Networking and Computing in Health Care Industry, Nancy, France, Jun. 2002.

[6] Recommentation H.263: Video Coding for Low Bit Rate Communication, ITU-T standard H.263, 1998.

[7] T. Wiegand, G. J. Sullivan, G. Bjontegaard, and A. Luthra, "Overview of the H.264/AVC video coding standard," IEEE Trans. Image Process., vol. 13 , no. 7, pp. 560-576, Jul. 2003.

[8] D. S. Taubman and M. Marcellin, JPEG2000: Image Compression Fundamentals, Standards, and Practice. Norwell, MA: Kluwer, 2001

[9] Hawk Voice Application Programming Interface. [Online]. Available: http://www.hawksoft.com/hawkvoice

[10] J. loup Gailly and M. Adler. Zlib Version 1.4.4. Library. [Online]. Available: http://www.gzip.org/zlib

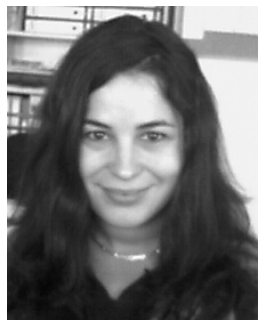

Cristina Cañero was born in Terrassa, Spain, on May 21, 1976. She received the B.Sc. degree in computer science engineering, the M.Sc. degree in computer vision, and the Ph.D. degree in computer science engineering from the Universitat Autonoma de Barcelona (UAB), Spain, in 1998, 2000, and 2003, respectively.

During her undergraduate studies, she worked for six months at the Universite Paris VIII, France, working on hand-written digits recognition, and participated in the Robocup'98. In 1998, she joined the Computer Science Department of the UAB as an Assistant Professor, teaching practical lessons of computer graphics. From 1999 to 2003, she was a Research Fellow at the Computer Vision Center in Barcelona, Spain, where she carried out research on deformable models for robust analysis and fusion of medical images. In 2003, and during one year, she was a Marie Curie Postdoctoral Fellow at the Informatics and Telematics Institute, Thessaloniki, Greece, working on networked virtual haptic environments and medical image processing. Since 2004, she has been Project Manager in the Computer Vision Center, Barcelona, Spain. Her current research interests include three-dimensional reconstruction and calibration, multimodal medical image fusion, and computer-assisted surgery.

Dr. Cañero is a Member of the Catalan Association for Artificial Intelligence, and a Reviewer of the IEEE TRANSACTIONS ON IMAGE PROCESSING.

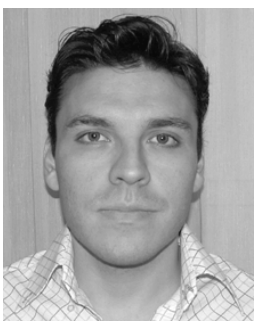

Nikolaos Thomos (S'02) was born in Cologne, Germany. He received the Diploma in electrical and computer engineering from Aristotle University of Thessaloniki, Thessaloniki, Greece, in 2000. He is currently working toward the Ph.D. degree at the same university.

He holds research and teaching assistantship positions in the Electrical and Computer Engineering Department, Aristotle University of Thessaloniki. $\mathrm{He}$ is also a Graduate Research Assistant with the Informatics and Telematics Institute, Thessaloniki, Greece. His research interests include image and video coding/transmission, multimedia networking, wavelets, and digital filters.

Mr. Thomos is a Member of the Technical Chamber of Greece.

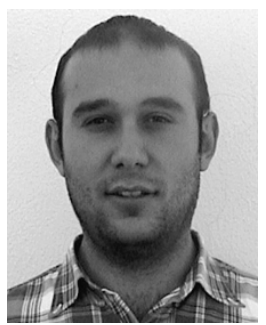

George A. Triantafyllidis was born in Thessaloniki, Greece, in 1975. He received the Diploma degree and the Ph.D. in electrical engineering from Aristotle University of Thessaloniki, Thessaloniki, Greece, in 1997 and 2002, respectively.

$\mathrm{He}$ is a Senior Researcher in the Informatics and Telematics Institute of Thessaloniki. Prior to his current position, he was a Researcher at the Aristotle University of Thessaloniki. His main research interests include image compression and analysis, 3-D data processing, medical image communication, and stereo and multiview image sequence coding. His involvement with those research areas has led to the coauthoring of eight papers in refereed journals and more than 30 papers in international conferences.

Dr. Triantafyllidis is a member of the Technical Chamber of Greece. 


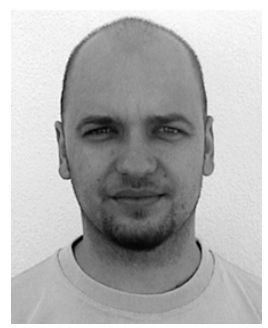

George C. Litos was born in Naoussa, Greece, in 1973.

He has ten years of professional experience in software development related to computer imaging, compression, communications, and robotics with applications to video, audio, compression, and health. Since 2000 , he is cooperating with the Informatics and Telematics Institute, Thessaloniki as a Research Associate. He has participated in several research projects funded by the European Union and the Greek Secretariat of Research and Technology.

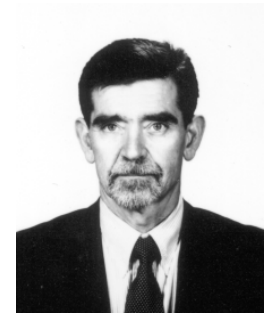

Michael Gerassimos Strintzis (S'68-M'70SM'80-F'04) received the Diploma in electrical engineering from the National Technical University of Athens, Athens, Greece, in 1967, and the M.A. and $\mathrm{Ph} . \mathrm{D}$. degrees in electrical engineering from Princeton University, Princeton, NJ, in 1969 and 1970, respectively.

He joined the Electrical Engineering Department at the University of Pittsburgh, Pittsburgh, PA, where he served as an Assistant Professor from 1970 to 1976, and an Associate Professor from 1976 to 1980. During that time, he worked in the area of stability of multidimensional systems. Since 1980, he has been a Professor of Electrical and Computer Engineering at the Aristotle University of Thessaloniki, Thessaloniki, Greece. $\mathrm{He}$ has worked in the areas of multidimensional imaging and video coding. Over the past ten years, he has authored over 100 journal publications and over 200 conference presentations. In 1998, he founded the Informatics and Telematics Institute, currently part of the Center of Research and Technology Hellas, Thessaloniki.

Dr. Strintzis was awarded one of the Centennial Medals of the IEEE in 1994 and the Empirikeion Award for Research Excellence in Engineering in 1999. 